\title{
PERFIL DA LESÃO RENAL AGUDA NOS CANDIDATOS AO TRANSPLANTE CARDÍACO EM UM HOSPITAL DE REFERÊNCIA
}

\author{
Profile of injury acute renal in candidates to heart transplantation in a reference hospital
}

\author{
Nasser Câmara Magalhães', Lenise Castelo Branco Camurça Fernandes², Paula Frassinetti Castelo Branco Camurça Fernandes³, \\ Ítalo Martins De Oliveira ${ }^{4}$, Joana Tássia Pinheiro De Figueiredo ${ }^{1}$, João David De Souza Neto ${ }^{5}$. Fernando Bacal', \\ Joana Thayne Pinheiro de Figueiredo7; Gabriel Moreira Furtado de Queiroz ${ }^{7}$
}

\section{RESUMO}

Introdução: A lesão renal aguda (LRA) é uma perda da função renal súbita e potencialmente fatal. Tem alta prevalência em pacientes com insuficiência cardíaca (IC) em estágio avançado, podendo, inclusive, limitar a eficácia do tratamento, aumentando a morbimortalidade em candidatos ao transplante cardíaco. Objetivo: Analisar o perfil clínico e epidemiológico da LRA em uma amostra de pacientes candidatos ao transplante cardíaco no Ceará. Método: Estudo descritivo transversal realizado no Hospital Dr. Carlos Alberto Studart Gomes, no período entre dezembro de 2011 e abril de 2013, avaliando-se LRA em 24 pacientes à espera de transplante cardíaco, utilizando os critérios de Acute Kidney Injury Network (AKIN). Resultados: A prevalência da LRA nessa série foi de $25 \%$, sendo quatro no estágio I, um no estágio II e um no estágio III de AKIN. Discussão: A associação da lesão renal com insuficiência cardíaca é devida a uma interação cardiorrenal complexa, denominada síndrome cardiorrenal. No estadiamento da doença renal crônica, pacientes com taxa de filtração glomerular (TFG) $<60 \mathrm{ml} / \mathrm{min} / 1.73 \mathrm{~m} 2$ têm maior risco de mortalidade. A LRA também é marcador de mau prognóstico, sendo necessária a aplicação dos critérios de AKIN para diagnóstico precoce. Conclusão: Devido à alta morbimortalidade da LRA em pacientes com insuficiência cardíaca grave e potencialmente instáveis, o diagnóstico precoce e o tratamento adequado são essenciais para melhor prognóstico dos pacientes.

Descritores: Lesão Renal Aguda; Síndrome Cardiorrenal; Transplante Cardíaco.

Instituições:

${ }^{1}$ Curso Acadêmico de Medicina - UECE - Fortaleza/CE

2 Depto. de Transplante do Hospital de Messejana Dr. Carlos Alberto Studart Gomes em convênio com o InCor-USP - Fortaleza/CE

${ }^{3}$ Curso de Medicina da UECE. Fortaleza, Ceará, Brasil.

${ }^{4}$ Coordenadoria do DINTER no Hospital de Messejana Dr. Carlos Alberto Studart Gomes - Fortaleza/CE

${ }^{5}$ Coordenadoria da Unidade de Transplante e Insuficiência Cardíaca do Hospital de Messejana Dr.Carlos Alberto Studart Gomes. Fortaleza/ CE

${ }^{6}$ Diretoria da Unidade Clínica de Transplante Cardíaco do InCor HCFMUSP - São Paulo/SP

${ }^{7}$ Curso Acadêmico de Medicina - Universidade Nilton Lins (UNL). Manaus, Amazonas, Brasil.

Correspondência:

Nasser Câmara Magalhães

Rua Carlos Vasconcelos N¹70/501 - CEP: 60115-170 - Fortaleza/CE

Tel.: (85) 9610-7374

E-mail: nasserkm@gmail.com

Recebido em: 09/04/2013

Aceito em: 30/01/2014

\section{INTRODUÇÃO}

A lesão renal aguda (LRA) representa a perda da função renal de maneira súbita e potencialmente reversível. ${ }^{1}$ A definição atual é baseada na creatinina e no débito urinário. Diferente da doença renal crônica (DRC) que é baseada na taxa de filtração glomerular (TFG), estimada pela depuração de creatinina por diferentes fórmulas. ${ }^{2}$

A prevalência de insuficiência renal em pacientes ambulatoriais com insuficiência cardíaca (IC) pode chegar a $29,6 \% .^{3}$ A presença de insuficiência renal pode limitar a eficácia do tratamento da IC, podendo, em casos mais graves, até requerer diálise, ${ }^{4}$ o que aumentaria a morbidade e os custos do tratamento.

A maioria dos pacientes com IC, com gravidade suficiente para necessitar de um transplante cardíaco, tem algum grau de alteração da função renal, que pode precipitar uma descompensação da IC, mas que também pode melhorar significativamente após o transplante cardíaco. ${ }^{1,4}$ 
A presente investigação tem por objetivo analisar a prevalência da LRA em uma amostra de pacientes à espera de transplante cardíaco no Ceará, correlacionando com o perfil clínico e epidemiológico da IC e suas causas e com o perfil da doença renal crônica que apresentam.

\section{MÉTODO}

Trata-se de um estudo descritivo e transversal. A população estudada corresponde a uma amostra de 24 pacientes do Hospital Dr. Carlos Alberto Studart Gomes, referência no estado do Ceará, internados ou em tratamento ambulatorial, que correspondiam aos critérios de inclusão, ou seja, ter idade superior a 18 anos, já estar na lista de espera para transplante cardíaco e concordar com o termo de consentimento livre e esclarecido (TCLE), no período entre dezembro de 2011 e abril de 2013.

As variáveis avaliadas correspondem ao sexo, idade, índice de massa-corpórea (IMC), dosagem dos níveis séricos de vitamina $D$ (Vit. D), creatinina, fração de ejeção (FE), classe funcional da IC, causa da IC, mensuradas logo após o paciente ter sido incluído na lista de espera e coletadas em um formulário previamente estabelecido. Elas foram analisadas estatisticamente pela frequência absoluta, relativa e média, sendo definido o perfil de prevalência da DRC e LRA nesses pacientes.

A DRC foi estadiada em níveis de TFG, estimadas por três fórmulas: Cockcroft-Gault (CG), Modification Of Diet In Renal Disease Study (MDRD) e Chronic Kidney Disease Epidemiology Collaboration (CKD-EPI). 5 A LRA foi definida e classificada de acordo com os critérios da Acute Kidney Injury Network (AKIN): I - elevação da creatinina sérica superior a $0,3 \mathrm{mg} / \mathrm{dL}$ ou aumento de uma vez e meia a duas vezes o valor basal; II - elevação da creatinina sérica de duas a três vezes o valor basal; III - elevação da creatinina sérica superior a $0,5 \mathrm{mg} / \mathrm{dL}$ em pacientes com creatinina superior a 4 ou aumento de três vezes o valor basal. ${ }^{6,7}$

A apresentação dos resultados foi de forma tabular e gráfica. A tabulação e a análise dos resultados foram realizadas por meio de planilhas e gráficos no programa do Microsoft Office Excel 2007.

O projeto de pesquisa respeitou a Declaração de Helsinque sobre Ética em Pesquisa com Seres Humanos e os princípios da Beneficência, da Não Maleficência e da Autonomia e seguiu as recomendações da Resolução 196/96 do Conselho Nacional de Saúde. Este trabalho foi realizado mediante aprovação pelo comitê de ética em pesquisa do Hospital Dr. Carlos Alberto Studart Gomes com o Processo de No 823-11. Adicionalmente, os pesquisadores envolvidos nesse trabalho não apresentam conflito de interesse.

\section{RESULTADOS}

Foram avaliados 24 pacientes e, após a análise do perfil clínico-epidemiológico (Tabela 1), foi mostrado que destes, a maioria eram homens (21), a média de idade 50,1 anos (Desvio padrão=10,7) a média geral de IMC 24,72 (Desvio padrão=2,98) e a média de FE $28,08 \%$ (Desvio padrão=3,93).

A distribuição das causas de IC na amostra foi: idiopática com seis pacientes, correspondendo a $25 \%$, seguida de IC isquêmica com quatro $(16,7 \%)$, chagásica com quatro $(16,7 \%)$ e alcoólica com quatro $(16,7 \%)$, um $(4,2 \%)$ com miocardiopatia dilatada, orovalvar com dois $(8,3 \%)$, um $(4,2 \%)$ com hipertensiva e outras causas com um $(4,2 \%)$.

New York Heart Association (NYHA), mostrou que a maioria dos pacientes encontrava-se em classe funcional (CF) III quinze $(58,3 \%)$ e Desvio padrão=1,35, seguida de CF II cinco $(20,9 \%)$ e desvio padrão $=0,94$, CF IV três (12,5\%) e desvio padrão=0, e CF I um (4,2\%) e desvio padrão $=0$.

Tabela 1 - Perfil clínico-epidemiológico dos pacientes avaliados

\begin{tabular}{|c|c|c|c|c|c|c|c|c|c|}
\hline Causa IC & N (\%) & Idade & $\begin{array}{c}\text { Sexo } \\
\text { Masculino }\end{array}$ & $\begin{array}{c}\text { IMC } \\
(\mathrm{Kg} / \mathrm{m} 2)\end{array}$ & $\begin{array}{l}\text { Fração de } \\
\text { ejeção }\end{array}$ & CF I & CF II & CF III & CF IV \\
\hline 1-Idiopática & $6(25 \%)$ & 42 & $6(100 \%)$ & 24,75 & $27,80 \%$ & & 1 & 4 & 1 \\
\hline 2-Isquêmica & $4(16,7 \%)$ & $64, q 25$ & $4(100 \%)$ & 28,09 & $34,50 \%$ & & & 4 & \\
\hline 3-Chagásica & $4(16,7 \%)$ & 45,7 & $3(75 \%)$ & 21,8 & $21,80 \%$ & 1 & 3 & & \\
\hline 4-Dilatada & $2(8,3 \%)$ & 65,5 & $1(50 \%)$ & 22,23 & $25,50 \%$ & & & 1 & 1 \\
\hline 5-Alcoólica & $4(16,7 \%)$ & 46,5 & $4(100 \%)$ & 25,21 & $26,50 \%$ & & 1 & 3 & \\
\hline 6-Hipertensiva & $2(4,2 \%)$ & 62 & $1(100 \%)$ & 29,19 & 28,00 & & & 1 & \\
\hline 7-Orovalvar & $2(8,3 \%)$ & 36 & $2(100 \%)$ & 25,3 & $27,50 \%$ & & & 1 & 1 \\
\hline 8-Outras & $1(4,2 \%)$ & 60 & $0(0 \%)$ & 20,33 & $33 \%$ & & & 1 & \\
\hline
\end{tabular}

IMC = Índice de massa corpórea

$\mathrm{CF}=$ Classe funcional 
Em relação à avaliação da DRC, os pacientes foram estratificados quanto ao clearance de creatinina, segundo as fórmulas: CG, mostrando que sete pacientes tinham uma TFG $<60 \mathrm{ml} / \mathrm{min} / 1,73 \mathrm{~m}^{2}$ (Tabela 2); MDRD e CKD-EPI (Tabela 3). Apesar das diferenças nos valores absolutos das depurações calculadas, os resultados foram semelhantes quanto ao número de pacientes em cada estádio de classificação, sendo sete $(29,2 \%)$ pacientes com TFG $<60 \mathrm{ml} / \mathrm{min} / 1,73 \mathrm{~m}^{2} .{ }^{5}$

Avaliou-se ainda, em conjunto com o estadiamento pelo método MDRD e CKD-EPI, a correlação dos estágios de $\mathrm{DRC}$ com os valores de IMC, idade e vit. D. Percebeu-se que não existia relação direta entre o estágio de DRC e a média de IMC dos pacientes, mas sendo possível inferir que a maioria dos pacientes avaliados eram eutróficos, sendo que apenas o estágio 3a teve média de IMC com sobrepeso. Quanto à idade, também não houve relação direta, porém, em todos os estágios, a média foi maior que 40 anos, correspondendo ao esperado de a IC ser mais prevalente em faixas etárias maiores. Já na Vit. $D$, foi mostrado que, para os pacientes com TFG $\geq 60 \mathrm{ml} /$ $\mathrm{min} / 1,73 \mathrm{~m} 2$, a vit. D estava na faixa de normalidade ( $>30 \mathrm{ng} /$ $\mathrm{ml})$. No entanto, para os de TFG $<60 \mathrm{ml} / \mathrm{min} / 1,73 \mathrm{~m} 2$, a vit. $\mathrm{D}$ encontrava-se insuficiente $(<30 \mathrm{ng} / \mathrm{ml})$ (Tabela 3$)$.

Tabela 2 - Classificação com Base em Estágio da Doença Renal Crônica pelo método Cockcroft-Gault

\begin{tabular}{lccc}
\hline $\begin{array}{c}\text { Estágios de função } \\
\text { renal }\end{array}$ & $\mathbf{N}(\%)$ & $\begin{array}{c}\text { Creatinina } \\
(\mathbf{m g} / \mathbf{d L})\end{array}$ & $\begin{array}{c}\text { CG } \\
(\mathbf{m l} / \mathbf{m i n} / \mathbf{1}, 73 \mathbf{m} 2)\end{array}$ \\
\hline Estágio 1 (TFG>90) & $4(16,7 \%)$ & 0,7 & 116,15 \\
Estágio 2 (TFG 60-89) & $13(54,2 \%)$ & 1,21 & 75,65 \\
Estágio 3a (TFG 45-59) & $4(16,7 \%)$ & 1,47 & 52,75 \\
Estágio 3b (TFG 30-44 & $2(8,3 \%)$ & 1,75 & 37,27 \\
Estágio 4 (TFG 14-29) & $1(4,2 \%)$ & 3,1 & 15,65 \\
\hline
\end{tabular}

DRC $=$ Doença Renal Crônica

$\mathrm{CG}=$ Método Cockcroft Gault
A avaliação da prevalência de LRA no serviço de transplante cardíaco do Hospital Dr. Carlos Alberto Studart Gomes mostrou que seis pacientes (25\%) apresentaram algum grau dessa complicação, sendo quatro no grau I $(16,7 \%)$, um no grau II $(4,2 \%)$ e um no grau III $(4,2 \%)$ (Tabela 4$)$. Sendo avaliada a correlação da LRA com a idade, FE e Vit.D, sendo que relações diretas não foram observadas entre idade e FE com LRA. Já a LRA graus II e III tiveram maior associação com insuficiência de Vit. D.

Tabela 4 - Classificação de lesão renal aguda nos pacientes avaliados segundo os critérios da Acute Kidney Injury Network

\begin{tabular}{lccccc}
\hline $\begin{array}{c}\text { Lesão renal } \\
\text { aguda } \\
\text { AKIN }\end{array}$ & N (\%) & $\begin{array}{c}\text { Creatinina } \\
(\mathbf{m g} / \mathbf{d L})\end{array}$ & $\begin{array}{c}\text { Idade } \\
(\text { anos) }\end{array}$ & $\begin{array}{c}\text { Fração de } \\
\text { ejeção (\%) }\end{array}$ & $\begin{array}{c}\mathbf{2 5 - ( O H )} \\
\text { Vit.D } \\
(\mathbf{n g} / \mathbf{m l})\end{array}$ \\
\hline Sem LRA & $18(75 \%)$ & 1,08 & 52,6 & $28,20 \%$ & 32,4 \\
I & $4(16,7 \%)$ & 1,59 & 39 & $24,80 \%$ & 30,27 \\
II & $1(4,2 \%)$ & 2,12 & 39 & $26 \%$ & 22,6 \\
III & $1(4,2 \%)$ & 3,1 & 60 & $33 \%$ & 16,8 \\
\hline
\end{tabular}

LRA = lesão renal aguda

AKIN = Acute Kidney Injury Network

\section{DISCUSSÃO}

Grande parte dos pacientes com IC tem alteração da função renal. ${ }^{1,4} \mathrm{Na}$ maioria ocorre recuperação da função renal após o transplante cardíaco, pois a disfunção renal é secundária à isquemia renal crônica consequente à baixa perfusão renal. 8 Contudo, nem todas as alterações na função renal vão melhorar simplesmente com a melhora da IC, pois lesões já ocorreram. ${ }^{9}$

Tabela 3 - Classificação com Base em Estágio da Doença Renal Crônica pelo método Modification Of Diet In Renal Disease Study (MDRD) e Chronic Kidney Disease Epidemiology Collaboration (CKD-EPI)

\begin{tabular}{|c|c|c|c|c|c|c|c|}
\hline $\begin{array}{l}\text { Estágios de } \\
\text { função renal }\end{array}$ & N (\%) & $\begin{array}{c}\text { Creatinina } \\
(\mathrm{mg} / \mathrm{dL})\end{array}$ & $\begin{array}{c}\text { MDRD } \\
\left(\mathrm{ml} / \mathbf{m i n} / \mathbf{1}, 73 \mathbf{m}^{2}\right)\end{array}$ & $\begin{array}{c}\text { CKD-EPI } \\
\left(\mathrm{ml} / \mathbf{m i n} / \mathbf{1}, 73 \mathbf{m}^{2}\right)\end{array}$ & $\underset{\left(\mathbf{k g} / \mathbf{m}^{2}\right)}{\mathrm{IMC}}$ & $\begin{array}{l}\text { Idade } \\
\text { (anos) }\end{array}$ & $\begin{array}{c}25-(\mathrm{OH}) \\
\text { Vit.D (ng/ml) }\end{array}$ \\
\hline $\begin{array}{l}\text { Estágio } 1 \\
(\mathrm{TFG}>90)\end{array}$ & $6(25 \%)$ & 0,76 & 119,33 & 112,83 & 22,3 & 40,6 & 33,25 \\
\hline $\begin{array}{l}\text { Estágio } 2 \\
\text { (TFG (60-89) }\end{array}$ & $9(37,5 \%)$ & 1,14 & 69,77 & 70,77 & 24,7 & 54,2 & 36,03 \\
\hline $\begin{array}{l}\text { Estágio 3a } \\
\text { (TFG 45-59 }\end{array}$ & $6(25 \%)$ & 1,49 & 52,5 & 52,5 & 28 & 54,8 & 24,45 \\
\hline $\begin{array}{l}\text { Estágio 3b } \\
\text { (TFG 30-44) }\end{array}$ & $2(8,3 \%)$ & 2,06 & 38,5 & 39 & 24,7 & 41 & 28,2 \\
\hline $\begin{array}{l}\text { Estágio } 4 \\
\text { (TFG 14-29) }\end{array}$ & $1(4.250$ & 3,1 & 16 & 16 & 20,3 & 60 & 16,8 \\
\hline
\end{tabular}

DRC $=$ Doença Renal Crônica

MDRD = Modification Of Diet In Renal Disease Study

CKD-EPI = Chronic Kidney Disease Epidemiology Collaboration 
A disfunção renal no contexto da IC está associada a uma interação cardiorrenal complexa. ${ }^{4}$ Essa frequente associação chama-se síndrome cardiorrenal (SCR) 2,10 e tem importantes implicações terapêuticas e prognósticos. ${ }^{11}$

A síndrome cardiorrenal decorre das alterações hemodinâmicas da insuficiência cardíaca, que promove ativação de vias neuro-hormonais, inicialmente compensatórias, mas que podem progressivamente tornar-se prejudiciais. ${ }^{10}$ Além da hiperatividade simpática, ativação do sistema renina-angiotensina-aldosterona, mediadores inflamatórios, estresse oxidativo, disfunção endotelial, aterosclerose, proteinúria, anemia, caquexia, insuficiência de vit. D. ${ }^{10}$ Esta última, avaliada na presente investigação, mostrou que há associação para pacientes com TFG $<60 \mathrm{ml} / \mathrm{min} / 1,73 \mathrm{~m} 2$ na DRC e em graus II e III (Tabela 3).

No contexto dos pacientes com IC, os tipos mais importantes de SRC são os tipos 1 e 2.

$\mathrm{Na}$ SCR tipo 1, em que uma rápida piora da função cardíaca leva à LRA, a lesão renal parece ser mais grave em pacientes com redução da fração de ejeção (FE) ${ }^{3,11}$ e sua presença afeta a evolução do paciente, podendo inibir o uso de drogas que aumentam a sobrevida na IC. ${ }^{11}$

É comum ainda na hospitalização por IC descompensada, podendo ocorrer em $25-50 \%$ dos pacientes, dependendo da série de casos, sendo um marcador de pior prognóstico. ${ }^{12,13}$ Este estudo não mostrou correlação entre a gravidade da LRA e a FE.

Na SCR tipo 2, em que anormalidades crônicas na função cardíaca levam à DRC progressiva, não há qualquer evidência consistente de associação entre a FE e a TFG,11 sendo altamente prevalente entre os pacientes com IC, chegando a cerca de $50 \%$ apresentar TFG $<60$ $\mathrm{ml} / \mathrm{min} / 1.73 \mathrm{~m}^{2}$, níveis de TFG, a partir dos quais o risco de mortalidade aumentou significativamente. . $^{3,9,11,12}$ Portanto, esse tipo é a provável causa da TFG $<60 \mathrm{ml} /$ $\mathrm{min} / 1,73 \mathrm{~m}^{2}$ nos pacientes avaliados, sete pelo CG e nove pelo MDRD e CKD-EPI.
O MDRD pode ser mais preciso em pacientes com menor TFG, enquanto o CG é mais preciso em pacientes com DRC mais leve, ${ }^{14}$ o que pode explicar as discrepâncias no estadiamento da DRC com essas diferentes fórmulas, além do maior número de pacientes com TFG inferior a $60 \mathrm{ml} / \mathrm{min} / 1.73 \mathrm{~m}^{2}$ quando comparada às fórmulas MDRD (nove pacientes) e o CG (sete pacientes) neste estudo.Os níveis de creatinina são afetados pela massa muscular, a qual pode ser substancialmente reduzida na caquexia cardíaca. Por isso, as fórmulas desenvolvidos para estimar a TFG são lentas para detectar lesão renal precoce,${ }^{14}$ tendo importância a utilização de algum dos critérios específicos para LRA. Os estudos mostraram que não há superioridade clara entre os critérios RIFLE e AKIN para o estabelecimento de LRA, porém o índice AKIN pode ser preferível, já que possui a capacidade de detectar LRA mais cedo, justamente por levar em conta valores menores dos biomarcadores. ${ }^{7,15,16}$

A LRA é marcador de mau prognóstico em pacientes com IC crônica, ${ }^{3}$ como no caso dos candidatos ao transplante cardíaco, podendo influenciar no tratamento da IC, predispondo a: menor eficácia do tratamento do paciente descompensado, intoxicação medicamentosa pelas drogas utilizadas na compensação do paciente com IC, diálise ou mesmo óbito do paciente.

\section{CONCLUSÃO}

Visto a alta prevalência da LRA no paciente com IC, principalmente do paciente com IC na lista de espera do transplante cardíaco, que normalmente está mais grave e debilitado, a identificação e tratamento adequado dos pacientes cardiorrenais são essenciais..$^{8,12}$

Portanto, o esforço para preservar a função renal é de extrema importância em pacientes com IC crônica..$^{12} \mathrm{~A}$ abordagem multidisciplinar da SCR ajuda a melhorar o prognóstico desses pacientes ${ }^{11}$ e a colaboração ativa de nefrologistas nas decisões terapêuticas dos pacientes cardiorenais em departamentos de cardiologia é necessária e benéfica. ${ }^{12}$ 


\section{ABSTRACT}

Introduction: Acute kidney injury (AKI) is a loss of sudden and potentially fatal kidney function. It has a high prevalence in patients with heart failure $(\mathrm{HF})$, and may also limit the effectiveness of treatment, increasing morbidity and mortality. Objectives: To analyze the clinical and epidemiological profile of acute kidney injury in a sample of patients eligible for cardiac transplantation in Ceará. Methods: Cross-sectional descriptive study conducted at Hospital Dr. Carlos Alberto Gomes Studart, between December 2011 and April 2013, evaluating LRA in 24 patients awaiting heart transplantation, using the Acute Kidney Injury Network (AKIN) criteria. Results: The prevalence of acute kidney injury in this series was $25 \%$, four in stage I, one in stage II and stage III of the AKIN. Discussion: The association of renal injury with heart failure is due to a complex cardiorenal interaction called cardiorenal syndrome. The staging of chronic kidney disease, patients with glomerular filtration rate GFR $<60 \mathrm{ml} / \mathrm{min} / 1.73 \mathrm{~m} 2$ have a higher risk of mortality. The LRA also is bad prognostic marker, the application requiring the AKIN criteria for early diagnosis. Conclusion: Due to high morbidity and mortality of acute kidney injury in this group of patients with severe heart failure and potentially unstable, early diagnosis and appropriate treatment is essential for better prognosis.

Keywords: Acute Kidney Injury, Cardio-Renal Syndrome, Heart Transplantation.

\section{REFERÊNCIAS}

1. Costa JAC, Moyses-Neto M, Vieira-Neto OM. Insuficiência renal aguda na terapia intensiva. Medicina intensiva. 1998;31:532-51.

2. Triposkiadis F, Starling RC, Boudoulas H, Giamouzis G, Butler J. The cardiorenal syndrome in heart failure: cardiac? renal? syndrome?. Heart Failure Reviews. 2012;17(3):355-66.

3. Bocchi EA, Marcondes-Braga FG, Bacal F, Ferraz AS, Albuquerque D, Rodrigues DA, et al. Atualização da Diretriz Brasileira de Insuficiência Cardíaca Crônica - 2012. Arquivos Brasileiros De Cardiologia. 2012;98 Suppl:1-33.

4. Bocchi EA, Vilas-Boas F, Perrone S, et. al. I Diretriz LatinoAmericana para avaliação e conduta na Insuficiência Cardíaca Descompensada. Arquivos Brasileiros De Cardiologia. 2005;85 Suppl:1-48.

5. Bastos MG, Kirsztajn GM. Doença renal crônica: importância do diagnóstico precoce, encaminhamento imediato e abordagem interdisciplinar estruturada para melhora do desfecho em pacientes ainda não submetidos à diálise. J. Bras. Nefrol. 2011;33(1):93-108.

6. Mehta RL, Kellum JA, Shah SV, Molitoris BA, Ronco C, Warnock DG, et al. Acute Kidney Injury Network: report of an initiative to improve outcomes in acute kidney injury. Crit Care. 2007;11(2):R31

7. Valette $X$, du Cheyron D. A critical appraisal of the accuracy of the RIFLE and AKIN classifications in defining "acute kidney insufficiency" in critically ill patients. Crit Care. 2013 Apr;28(2):116-25.

8. Bacal F, Souza-Neto JD, Fiorelli AI, Mejia J, Marcondes-Braga FG, Mangini S, et. al. II Diretriz Brasileira de Transplante Cardíaco. Arquivos Brasileiros De Cardiologia. 2009; 94 Suppl(1):16-73.
9. Udani SM, Koyner JL. The effects of heart failure on renal function. Cardiology Clinics, 2010;28(3):453-65.

10.Hatamizadeh P, Fonarow GC, Budoff MJ, Darabian S, Kovesdy CP, Kalantar-Zadeh K. Cardiorenal syndrome: pathophysiology and potential targets for clinical management. Nature Reviews Nephrology. 2013 Feb;9(2):99-111.

11. Ronco C, Haapio M, House AA, Anavekar N, Bellomo R. Cardiorenal Syndrome. Journal of the American College of Cardiology. 2008; 52(19): 1527-39.

12. Waldum B, Os I. The Cardiorenal Syndrome: What the Cardiologist Needs to Know. Cardiology. 2013;126(3):175-86.

13 .Montera MW, Pereira SB, Colafranceschi AS, Almeida DR; Tinoco EM, Rocha RM, et al. Sumário de Atualização da II Diretriz Brasileira de Insuficiência Cardíaca Aguda 2009/2011. Arquivos Brasileiros De Cardiologia. 2012;98(5):375-83.

14. Cole RT, Masoumi A, Triposkiadis F, Giamouzis G, Georgiopoulou V, Kalogeropoulos A, et al. Renal dysfunction in heart failure. Medical Clinics of North America. 2012;96(5):95574.

15. Barros LCN, Silveira FS, Silveira MS, Morais TC, Nunes MAP; Bastos KA. Insuficiência renal aguda em pacientes internados por insuficiência cardíaca descompensada - Reincade. J. Bras. Nefrol. 2012;34(2):122-9.

16. Levi TM, Souza SP, Magalhães JG, Carvalho MS, Cunha ALB, Dantas JGAO, et al. Comparação dos critérios RIFLE, AKIN e KDIGO quanto a capacidade de predição de mortalidade em pacientes graves. Rev. Bras. Ter. Intensiva. 2013;25(4):290-6. 